\title{
The Optimal Weight Allocation Method of Containing Predicted Values and Measured Values
}

\author{
Yanting Wang ${ }^{\mathrm{a}}$, Xu Zhang, Fan Zhao, Min Liu and Guolin Liu \\ China Luoyang Electronic Equipment Testinh Center, Luoyang 471003, China; \\ awangyanting454799@163.com
}

\begin{abstract}
Keywords: Target tracking, Data fusion, the optimal weight allocation method, Maneuvering frequency.
\end{abstract}

\begin{abstract}
For the practical issue of multi-sensor data fusion in target tracking, this paper combines the predicted values and the measured values weighted together. The optimal weight allocation method of Containing predicted values and measured values is presented. In theory, the more the number of sensors is, the higher the precision of the data fusion will be. When the predicted value has been seen as a measured value of a sensor, the precision of data fusion will be improved. In addition, a method of the establishment of the maneuvering frequency which can get a better effect of tracking is presented. Numerical examples show that, the optimal weight allocation method of containing predicted values and measured values is better than the method of containing only measured values, the improvement on maneuvering frequency of current statistical model can obtain better tracking effect.
\end{abstract}

\section{Introduction}

With the rapid development of target tracking and information fusion technology people begin to integrate utilize the measurements from multi-sensor to estimate the position and kinetics parameters, and useful information is extract to the greatest extent to track the moving object. How to effective fuse the measurements from multi-sensor and obtain better tracking performance compared to the single sensor are an important research in multi-sensor target tracking domain ${ }^{[1]}$. Due to the position of the each sensor in the process of data fusion, the fusion of different methods, such as nearest neighbor method, the fusion method based on weights, the fusion method based on fuzzy inference and the fusion method based on neural network are presented ${ }^{[2 \sim 3]}$. In theory, the literature [1 2] proved that the more the number of sensors, the data fusion accuracy will be higher.

About the definition of data precision, generally considered that the data precision is the degree of precision accuracy of the data, but it is difficult to express the data precision of measured values with its "true value" proximity. Because the predicted values of data represents the true value to a certain extent ${ }^{[4]}$, so the predicted values and measured values are weighing in combination by the optimal weight allocation method. In this place, the predicted value is treated as a measured value of sensor. When a new measured value of sensor (predicted value) is added, the data fusion accuracy will be higher.

Firstly, due to the accuracy of target tracking is the basis of multi-sensor data fusion, this paper study the current statistical model. A method of the establishment of the maneuvering frequency which can get a better effect of tracking is presented. Then, for improving the robustness of tracking system, a robust method which can estimate the standard deviation of the predicted values and the measured values is established. Finally by an example, it shows that the improvement on maneuvering frequency of current statistical model can obtain better tracking effect, the optimal weight allocation method of containing predicted values and measured values is better than the method of containing only measured values. 


\section{The Improvement on Maneuvering Frequency of Current Statistical Model}

The current statistical model is presented by professor Zhou Hongren based on singer model which supposes the current acceleration of maneuvering targets obey the modified Rayleigh distribution ${ }^{[5]}$. The model can reflect the range and intensity changes of the target maneuvering and has been used as one of the most widely model.

The discrete state equation of the current statistical model.

$X(k+1)=\Phi(k+1, k) X(k)+U(k) \bar{a}+W(k)$

In this formula, $X(k)$ is the state of target, $W(k)$ is the state of system noise, $\bar{a}$ is the mean of acceleration, $\mathrm{T}$ is sampling period, $\alpha$ is the maneuvering frequency of current statistical model, $E(W(k))=0, Q(k)=E\left(W(k) W(k)^{\prime}\right)$,

$$
\Phi(k+1, k)=\left[\begin{array}{ccc}
1 & T & \frac{1}{\alpha^{2}}\left(-1+\alpha T+e^{-\alpha T}\right) \\
0 & 1 & \frac{1}{\alpha^{2}}\left(1-e^{-\alpha T}\right) \\
0 & 0 & e^{-\alpha T}
\end{array}\right] \quad U(k)=\left[\begin{array}{c}
\frac{1}{\alpha^{2}}\left(-T+\frac{\alpha T^{2}}{2}+\frac{1-e^{-\alpha T}}{2}\right) \\
T-\frac{1}{\alpha}\left(1-e^{-\alpha T}\right) \\
1-e^{-\alpha T}
\end{array}\right]
$$

The Observation equation

$Z(k)=H(k) X(k)+V(k)$

In this formula, $Z(k)$ is the observation vector, $H(k)$ is the observation matrix, $E(V(k))=0$, $R(k)=E(V(k) V(k) ')$. Then, we can get the formula of kalman filtering.

$$
\begin{aligned}
& \hat{X}(k+1 \mid k+1)=\hat{X}(k+1 \mid k)+K(k+1)[Z(k+1)-H(k+1) \hat{X}(k+1 \mid k)] \\
& \hat{X}(k+1 \mid k)=\Phi(k+1, k) \hat{X}(k \mid k)+U(k) \bar{a} \\
& K(k+1)=P(k+1 \mid k) H^{\prime}(k+1)\left[H(k+1) P(k+1 \mid k) H^{\prime}(k+1)+R(k+1)\right]^{-1} \\
& P(k+1 \mid k)=\Phi(k+1, k) P(k \mid k) \Phi^{\prime}(k+1, k)+Q(k) \\
& P(k+1 \mid k+1)=[I-K(k+1) H(k+1)] P(k+1 \mid k)
\end{aligned}
$$

Because both $\Phi$ and Q contain $\alpha$, so if we change the value of $\alpha$, we can change both $\Phi$ and $\mathrm{Q}$ to make them close to the truely state of the target. In most of the literature, the maneuvering frequency of the target is adaptively adjusted according to the residuals of target tracking, such as distance function method, minimum mean square error algorithm, etc.

Literature [6] comes up with $d(k)$ as innovation vector for target.

$d(k)=Z(k)-H(k) X(k \mid k-1)$

In this formula, $d(k)$ is gaussian white noise process, and its covariance matrix is $S(k)$.

$S(k)=H(k) P(k \mid k-1) H^{\prime}(k)+R(k)$. If we define the distance function

$$
D(k)=d^{\prime}(k) S^{-1}(k) d(k)
$$

Then we can get $\alpha=\frac{D(k)}{M} \alpha, M$ is the threshold value.

In this paper, we consider the first-order difference vector of adjoining times

$$
d(k)=H(k) \hat{X}(k)-H(k-1) \hat{X}(k-1)
$$

In this formula, $\hat{X}(k)$ is the predicted value of $k$ time, $\hat{X}(k-1)$ is the predicted value of $k-1$ time. So we define the distance function

$D(k)=d^{\prime}(k) d(k)$ 
Because $D(k)$ represents the displacement of target in two adjacent moment, so we can get $\alpha(k)=\frac{D(k)}{D(k-1)} \alpha(k-1) . \alpha$ Is real-time update.

\section{The Principle of the Optimal Weight Allocation Method of Containing Predicted Values and Measured Values}

Suppose there are several sensors to measure a target and the number of sensors is $m, X_{i}$ is a measured value of $i$ sensor, $i=1,2, \cdots m$. By interference of kinds of random factors, $X_{i}$ is random. In actual measurement, suppose there are no system error for each sensor, $X_{i}$ obey normal distribution $N\left(\mu_{\text {真 }}, \sigma_{i}^{2}\right), \mu_{\text {真 }}$ is the true value of target, $\sigma_{i}^{2}$ is the variance of measured values . The larger the value of $\sigma_{i}^{2}$ is, the lower of the measuring precision of this sensor will be. Otherwise, the smaller the value of $\sigma_{i}^{2}$ is, the higher of the measuring precision of this sensor will be.

Calculating the weighted average of all the measured datas is called the weighted average method, the specific formula is as follows

$$
x_{r}=\sum_{i=1}^{m} \omega_{i} x_{i}
$$

In this formula, $\sum_{i=1}^{m} \omega_{i}=1$.

The definition of the weight value in optimal weight allocation method is

$$
\omega_{i}=\frac{1}{\sigma_{i}^{2} \sum_{j=1}^{m} \frac{1}{\sigma_{j}^{2}}}
$$

If the precision of the sensors is right, the optimal weight allocation method will be the best method for weight allocation.

Suppose $\hat{X}$ is the predicted value of the current target, we can obtain it by kalman filtering and it is independent with other sensors. $\hat{x}$ Obey normal distribution $N\left(\mu_{\text {真 }}, \hat{\sigma}^{2}\right), \mu_{\text {真 }}$ is the true value of target, $\hat{\sigma}^{2}$ is the variance of predicted value. Then $\hat{X}$ can be seen as a measured value which is measured by the $m+1$ sensor. According to the literature [2], the more the number of sensors is, the higher the precision of the data fusion will be. So when the predicted value is seen as a measured value, the precision of data fusion will be improved.

\section{Dynamic Estimation Method of Weights}

Suppose $x_{i}(k), i=1,2, \cdots m$ is a measured value of $i$ sensor in $k$ time, $\hat{x}(k)$ is the predicted value of target in $k$ time and $x_{r}(k)$ is the value after data fusion. Then we can step by step to get the dynamic estimation of the variance.

1) Calculate the error of measured value for each sensor

$\Delta x_{i}(k)=x_{i}(k)-\hat{x}(k), \quad i=1,2, \cdots m$

2) Calculate the mean error of the measured values

A general method for calculating the mean error of the measured values is that

$$
\Delta \bar{x}_{i}(k)=\frac{1}{N} \sum_{j=k-N+1}^{k} \Delta x_{i}(j), N \geq 5 \text {. }
$$


But this method is not robust. For getting a robust estimate and avoiding the influence of outliers, this paper will use the median estimate instead of the the mean estimate.

$\Delta \bar{x}_{i}(k)=$ median $\left(\Delta x_{i}(k), \Delta x_{i}(k-1), \cdots, \Delta x_{i}(k-N+1)\right)$

3) Calculate the variance of measured value for each sensor

$\sigma_{i}^{2}(k)=\frac{1}{N-1} \sum_{j=k-N+1}^{k}\left(\Delta x_{i}(k)-\Delta \bar{x}_{i}(k)\right)^{2}$

Now, we get the dynamic estimation of the variance for each sensor. Next, we will get the dynamic estimation of the variance for predicted value.

1) Calculate the error between predicted value and the value after data fusion

$\Delta \hat{x}(k-1)=\hat{x}(k-1)-x_{r}(k-1)$

2) Calculate the mean error of the predicted values

$$
\Delta \overline{\hat{x}}(k-1)=\operatorname{median}(\Delta \hat{x}(k-1), \Delta \hat{x}(k-2), \cdots, \Delta \hat{x}(k-N))
$$

3) Calculate the variance of predicted value

$$
\hat{\sigma}^{2}=\frac{1}{N-1} \sum_{j=k-N}^{k-1}(\Delta \hat{x}(k)-\Delta \overline{\hat{x}}(k))^{2}
$$

When we get the variance of the measured values and predicted values, we can use the optimal weight allocation method for fixed weight.

$$
\omega_{i}=\frac{1}{\sigma_{i}^{2}\left(\sum_{j=1}^{m} \frac{1}{\sigma_{j}^{2}}+\frac{1}{\hat{\sigma}^{2}}\right)}, \quad \omega_{m+1}=\frac{1}{\hat{\sigma}^{2}\left(\sum_{j=1}^{m} \frac{1}{\sigma_{j}^{2}}+\frac{1}{\hat{\sigma}^{2}}\right)}, \quad i=1,2, \cdots m
$$

When we get the weight of each data, we can get the value after data fusion.

$$
x_{r}=\sum_{i=1}^{m} \omega_{i} x_{i}+\omega_{m+1} \hat{x}
$$

In this formula, $\sum_{i=1}^{m+1} \omega_{i}=1$.

\section{Example Analysis}

For verifying the effect of data fusion, we choose the data that radar A and radar B track aircraft at the same time. The sampling rate of the radar is 20 frames per second and 5332 points are collected. We also have corresponding GPS data as the true value. Simplicity, we take coordinate y direction for validation.

1) The comparison between maneuvering frequency based on the changing of residual and maneuvering frequency based on the first-order difference vector of adjoining times.Figure 1 is the result of maneuvering frequency based on the changing of residual. Figure 2 is the result of maneuvering frequency based on the first-order difference vector of adjoining times. The graph's horizontal axis shows the time range and the vertical axis shows the difference between radar A data and GPS data. From the two figures, we can see that the improvement on maneuvering frequency of current statistical model can obtain better tracking. 


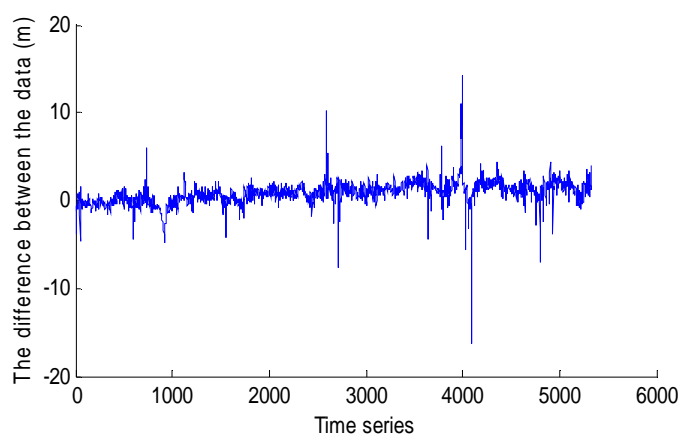

Fig. 1 Maneuvering frequency based on the changing of residual

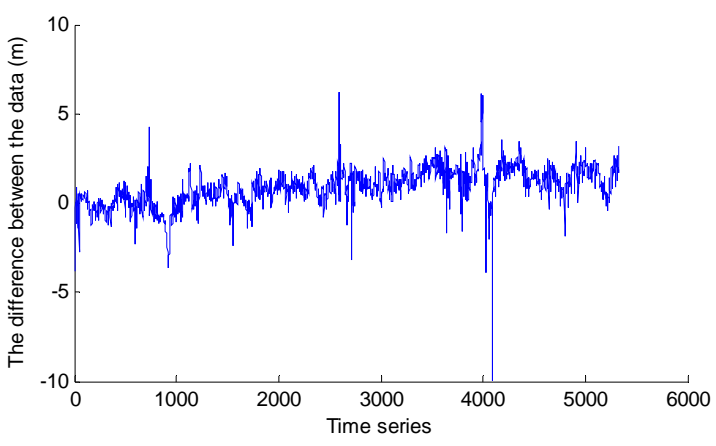

Fig. 2 Maneuvering frequency based on the first-order difference vector of adjoining times

2) The comparison between the optimal weight allocation method of Containing only measured values and the optimal weight allocation method of Containing predicted values and measured values.

Figure 3 is the variance after the measured values fusion. Figure 4 is the variance after the measured values and the predicted value fusion. From the two figures, we can see that the optimal weight allocation method of containing predicted values and measured values is better than the method of containing only measured values.

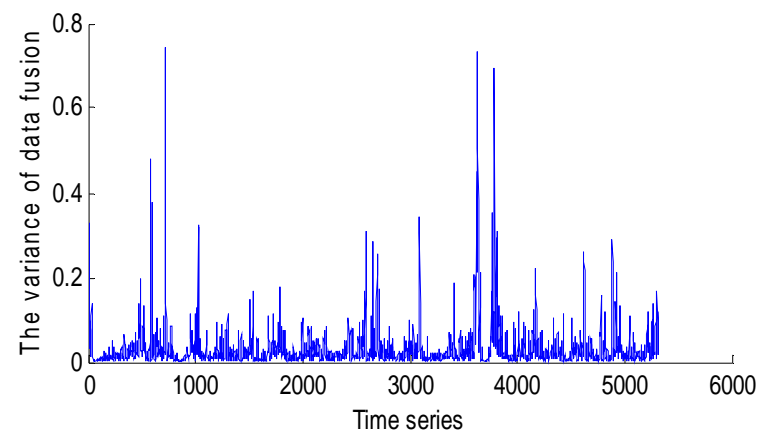

Fig. 3 The variance after the measured values fusion

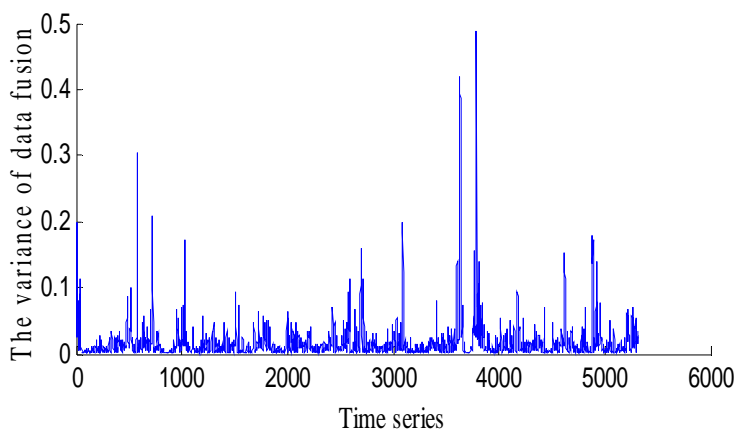

Fig. 4 The variance after the measured values and the predicted value fusion

3) The result analysis of data fusion. Figure 5 is the result of data fusion. The green line shows the difference between radar A data and GPS data, the bule line shows the difference between radar B data and GPS data, the red line shows the difference between data after data fusion and GPS data. From the figure, we can see that the data after data fusion avoides the influence of outliers and is better than any other measured data.

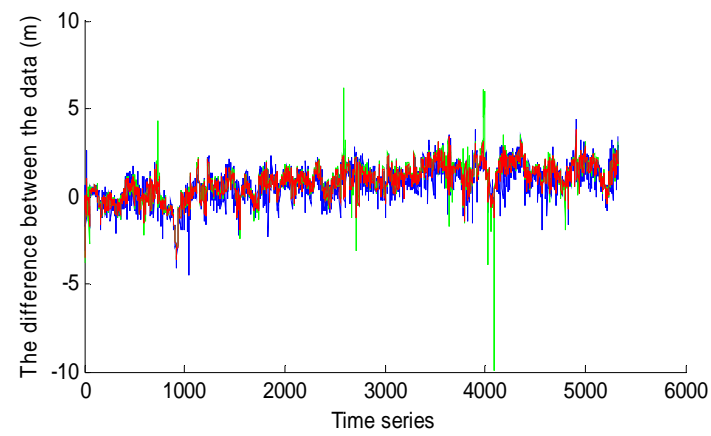

Figure 5. the result of data fusion

\section{Summary}

1) In this paper, a method of the establishment of the maneuvering frequency based on the first-order difference vector of adjoining times is used. By the method, we can obtain a better tracking. 
2) For getting a robust estimate and avoiding the influence of outliers, this paper uses the median estimate instead of the the mean estimate. When the datas contain lots of outliers, it will be useful.

3) The optimal weight allocation method of containing predicted values and measured values is better than the method of containing only measured values. Extraordinarily, when there are not lots of sensors, the method will be more useful and the precision of data fusion will be better.

\section{References}

[1]. Chui Bo.Key Technologies Research on Multi-sensor Data Fusion for Traget Tracking [D].Southwest Jiaotong University,2012.09.

[2]. Huang Youpeng.Research on Serveral Key Techniques of Multi-sensor Multi-Target Track correlation and Data Combination [D].Haebin Engineering University,2009.06.

[3]. Kang Yaohong. The theory and application of data fusion [M]. Xi'an University of Electronic Science and Technology Press, Xi'an, 2006.

[4]. Sui Lifen, Song Lijie, Cai Hongzhou. The basis of error theory and measurement adjustment [M]. Surveying and mapping press, Beijing, 2001.

[5]. Qian Huanming, Chen Liang, Man Guojing, et al. Adaptive tracking algorithm of maneuvering targets based on current statistical model[J].Syestem Engineering and Electronics,2011,10(10):2154-2158.

[6]. Ren Shaowei,Wang Rui,Zhang Pingding. An Adaptive Algorithm of Maneuvering Targets Tracking Based on Maneuvering Frequency [J]. Journal of Air Force Engineering University (Natural Science Edition) ,2004,5(1):32-35.

[7]. Xia Peilun,Wen Hong.Target tracking based on fuzzy in ference[J]. Electronics Optics \& Contral, 2003,10(1):23-27.

[8]. Li Qiuhua,Li Jicheng,Shen Zhenkang.Target tracking in the Dual Band IR Imageing Syestem Using Adeaptive weighting fusion Based on Fuzzy Inference[J].Journal of Electronics\&information Technology,2005,27(12):1922 -1926.

[9]. Liu Xinwang,Huang Wei. Fuzzy inf erence based optimization method for multiobjective decision making [J].Journal of Management Sciences In China, 2001, 12(4):71-76.

[10]. Lei Yang,Lei Jieying, et al. Techniques for target recognition based on adaptive intuitionistic fuzzy inference [J].Syestem Engineering and Electronics, 2010,7(7):1471-1475. 